\title{
Kinetic and Sensitivity Approach to the Mechanism of Inhibited Polymerization of Vinyl Acetate in the Presence of Furan Compounds.
}

\author{
Rebeca Vega and Jacques Rieumont
}

\begin{abstract}
The inhibited radical polymerization of vinyl acetate initiated by 2,2-azobisisobutyronitrile was studied in the presence of some furan derivatives bearing a double bond on the side group. The modelling of such systems has permitted to estimate the values of the kinetic parameters for these inhibitors. A sensitivity analysis was performed to obtain a better insight into the mechanism; it appears that macroradical degradative transfer reaction to furan compounds is the most important reaction in comparison with propagation or primary radical degradative transfer.
\end{abstract}

Keywords: Modelling; sensitivity analysis; inhibited polymerization; furan derivatives

\section{Introduction}

Kinetic treatment of retarded radical polymerization was developed by Kice ${ }^{[1]}$, and modified by Atkinson ${ }^{[2]}$. Later $\mathrm{Deb}^{3}$ proposed to include both macro-radical and primary radical degradative transfer reaction as mechanistic complications and gave a mathematical formulation for the process. Tudös ${ }^{[4]}$ has also contributed to the mechanism of inhibition and considered several possibilities and in each case obtained the characteristic parameters of the inhibitor. All these treatments are limited to obtain the ratio of constants through complicated linearizations.

Since 1983 Rieumont and co-workers ${ }^{[5-8]}$ have been studying thoroughly the kinetic features of the retarded and inhibited polymerization of vinyl acetate in the presence of furan compounds. The classical approach was complemented by a sensitivity analysis in order to discriminate between the determining, nondetermining and neglected steps of the mechanism. The kinetic parameters have been estimated by modelling the experimental data using non-linear methods. Recently a combination of these techniques with the GEAR's algorithm to solve the system of the ordinary differential equations (ODE) has given good results ${ }^{[9]}$ and reliable sets of kinetic parameters have been obtained in spite of the statistical dependence between them due to the steady state condition.

Following this approach, the present paper deals with the application of a sensitivity technique and estimation of macroradical and primary radical degradative transfer, reinitiation and cross termination constants, to the inhibited radical polymerization of vinyl acetate in the presence of some furan

Rebeca Vega, Faculty of Chemistry - Havana University, Zapata and G, Havana 10400, Cuba, Jacques Rieumont, State University of the North Fluminense. Center of Science and Technology, Av. Alberto Lamego 2000 - Campos de Goytacazes, CEP 28015-620, Rio de Janeiro, RJ 
compounds with conjugated side groups not fully studied kinetically to date; the side groups enhance their radical scavenging properties. These techniques lead to a deeper insight on the mechanism of inhibition for these series of compounds.

\section{Experimental}

Furylacrolein [3-(2-furyl)-propenal], 5methylfuranacrylic acid [3(2-(5-methylfuryl))propenoic acid] and 2-furylacrylmorfoline amide [3-(2-furypropen-tetra-hydro-1,4-oxazinamide] were kindly supplied by the Organic Chemistry Laboratory. Furfurylidenacetone [4-(2-furyl)-3buten-2-one], and hydrofuramide (N,N'-difurfuryliden-2-furanmethanediamine) were synthesized according to literature ${ }^{[10]}$. All the compounds were purified by standard techniques and characterized by IR and NMR spectra. Ethyl acetate (BDH) and 2,2'-azobisisobutyronitrile (AIBN; WAQO) were also purified by standard techniques and vinyl acetate $(\mathrm{BDH})$ was purified by prepolymerization and doubly distillation.

All details of the polymerization reaction, and the results of dilatometric determinations have already been reported ${ }^{[7]}$.

\section{Model Equations}

The mechanism proposed by Deb ${ }^{[3]}$ for retarded radical polymerization was assumed. This mechanism includes not only macroradical but primary radical complications:

$$
\begin{array}{ll}
\text { Initiation } & \mathrm{I} \stackrel{\mathrm{kd}}{\longrightarrow} 2 \mathrm{R}^{*} 2 \mathrm{f}_{\mathrm{kd}}[\mathrm{I}] \\
& \mathrm{R}^{*}+\mathrm{M} \stackrel{\mathrm{ki}}{\longrightarrow} \mathrm{M}^{*} \quad \mathrm{k}_{\mathrm{i}}[\mathrm{R}][\mathrm{M}]
\end{array}
$$

Propagation $\mathrm{M}^{*}+\mathrm{M} \stackrel{\mathrm{kp}}{\longrightarrow} \mathrm{M}^{*} \quad \mathrm{k}_{\mathrm{p}}\left[\mathrm{M}^{*}\right][\mathrm{M}]$

Termination $\mathrm{M}^{*}+\mathrm{M}^{*} \stackrel{\mathrm{kt}}{\longrightarrow} \mathrm{P} \quad \mathrm{k}_{\mathrm{t}}\left[\mathrm{M}^{*}\right]\left[\mathrm{M}^{*}\right]$

Macroradical $\mathrm{M}^{*}+\mathrm{Z} \longrightarrow \mathrm{MZ}^{*} \quad \mathrm{k}_{\mathrm{trs}}\left[\mathrm{M}^{*}\right][\mathrm{Z}] \mathrm{k}_{\mathrm{trs}}$ Degradative Transfer

Macroradical $\mathrm{MZ}^{*}+\mathrm{M} \longrightarrow \mathrm{M}^{*} \quad \mathrm{k}_{\mathrm{is}}\left[\mathrm{MZ}^{*}\right][\mathrm{M}]$

Re-initiation

Cross $\mathrm{MZ}^{*}+\mathrm{M}^{*} \longrightarrow \mathrm{P} \quad \mathrm{k}_{\mathrm{rts}}\left[\mathrm{MZ}^{*}\right]\left[\mathrm{M}^{*}\right]$

Macroradical Termination

Microradical $\mathrm{R}^{*}+\mathrm{Z} \longrightarrow \mathrm{RZ}^{*} \quad \mathrm{k}_{\text {trs }}^{\prime}\left[\mathrm{R}^{*}\right][\mathrm{Z}]$

Degradative transfer
Microradical $\mathrm{RZ}^{*}+\mathrm{M} \longrightarrow \mathrm{M}^{*} \quad \mathrm{k}_{\text {is }}^{\prime}\left[\mathrm{RZ}^{*}\right][\mathrm{M}]$

Re-initiation

Microradical $\mathrm{RZ}^{*}+\mathrm{M}^{*} \longrightarrow \mathrm{P} \quad \mathrm{k}_{\mathrm{rts}}\left[\mathrm{RZ}^{*}\right]\left[\mathrm{M}^{*}\right]$

Cross termination

From this set of reactions can be derived the set of ODE for the sensitivity analysis and for modelling the inhibited polymerization (see Appendix).

\section{Sensitivity analysis}

A program written in FORTRAN-77 MS was implemented on an IBM- compatible microcomputer so as to calculate all the normalized coefficients $\sigma$ of the sensitivity matrix

$$
\sigma_{\mathrm{ij}}=\frac{\partial \ln \mathrm{Ci}}{\partial \ln \mathrm{kj}}
$$

where the $\mathrm{Ci}$ are the concentrations of the reactants, intermediaries and products, and $\mathrm{kj}$ the kinetic parameters. These coefficients are calculated using a direct technique according to the methodology given by Come ${ }^{[11]}$. The system of ODE for the mechanism is solved calling a subroutine with the Gear algorithm ${ }^{[12]}$. All the concentrations are calculated at the desired time by using a set of suitable kinetic parameters obtained by a first modelling of the experimental data.

A complete sensitivity matrix for the case of furylacrolein during the inhibition period is shown as an example in Table 1. In this matrix each

Table 1. Sensitivity matrix for the system vinyl acetate $(3.254 \mathrm{~mol} / \mathrm{L})$ AIBN (0.002 mol/L)- Furylacrolein at $10 \mathrm{~min}$.

\begin{tabular}{lccccccc}
\hline & $\mathrm{I}$ & $\mathrm{M}$ & $\mathrm{Z}$ & $\mathrm{M}^{*}$ & $\mathrm{R}^{*}$ & $\mathrm{MZ}^{*}$ & $\mathrm{RZ}^{*}$ \\
\hline $\mathrm{kd}$ & 0 & 0 & -0.1 & 1.0 & 1.0 & 1.0 & 1.0 \\
$\mathrm{ki}$ & 0 & 0 & 0.1 & 0.9 & -40.2 & 0.6 & -146.8 \\
$\mathrm{kp}$ & 0 & 0 & 0 & 0 & 0 & 0 & 0 \\
$\mathrm{kt}$ & 0 & 0 & 0 & 0 & 0 & 0 & 0 \\
$\mathrm{ktrs}$ & 0 & 0 & -0.1 & -6.2 & 0 & 1.0 & 0.8 \\
$\mathrm{kis}$ & 0 & 0 & 0 & 0.1 & 0 & -0.2 & -0.2 \\
$\mathrm{krts}$ & 0 & 0 & 0.1 & -3.0 & 0 & -15.0 & 0.8 \\
$\mathrm{k}$ 'trs & 0 & 0 & 0 & 0 & 0 & 0 & 1.0 \\
$\mathrm{k}^{\prime}$ is & 0 & 0 & 0 & 0 & 0 & 0 & -0.2 \\
$\mathrm{k}^{\prime} \mathrm{rts}$ & 0 & 0 & 0 & 0 & 0 & 0 & -5.0 \\
$\mathrm{f}$ & 0 & 0 & -0.1 & 1.0 & 1.0 & 1.0 & 1.0 \\
\hline
\end{tabular}


Table 2. Compacted sumatory of the sensitivity coefficients of the kinetic parameters for each system during the inhibition period

\begin{tabular}{l|c|c|c|c|c|c|c|c|c|c|c}
\hline Compound & $\mathrm{f}$ & $\mathrm{kd}$ & $\mathrm{ki}$ & $\mathrm{kp}$ & $\mathrm{kt}$ & $\mathrm{ktrs}$ & $\mathrm{kis}$ & $\mathrm{krts}$ & $\mathrm{k}$ 'trs & $\mathrm{k}$ 'is & $\mathrm{k}$ 'rts \\
\hline furylacrolein & 4 & 4 & 18 & 0 & 0 & 8 & 0.3 & 18.9 & 1.0 & 1.2 & 5.0 \\
& & & 8 & & & & & & & & \\
& 4 & 4 & $*$ & 0 & 0 & 20.1 & 0 & 35.2 & 1.0 & 0.1 & 6.3 \\
fur furylidenacetone & 4 & 4 & $*$ & 0 & 1.4 & 2.0 & 0 & 280 & 1.3 & 0 & 210 \\
furylacrylmorfolineamide & 4 & 4 & $*$ & 0 & 26 & 1 & 0.3 & 1.2 & 1.0 & 0 & 185 \\
5-methylfuranacrylic ac. & 4 & 4 & $*$ & 0 & 0 & 1.3 & 0 & 170 & 1.2 & 0 & 158 \\
\hline
\end{tabular}

* The sensitivity coefficient is higher than 1000 .

normalized coefficient was evaluated according to Equation 1 when the kinetic parameter is changed to zero. Then if all the coefficients for a parameter were found to be zero or less than 0,01 , the reaction step can be considered negligible and ruled out of the mechanism.

The sensitivity matrix for all the systems under study was evaluated twice, first during the inhibition period and later during the normal polymerization. All the results are shown in a compacted form in tables 2 and 3. Each number represents the sum of the row vector in the matrix corresponding to each inhibitor.

Several overall conclusions can be drawn from tables 2 and 3 :

-The propagation and normal termination of the polymerizations can be considered in some cases negligible during the inhibition period, nevertheless they cannot be ruled out of the mechanism because a "normal" polymerization begins after the inhibitor is consumed and their weighting increases after the inhibition period.

-The cross terminations are the most important way of termination during the inhibition period.

- The primary complications cannot be ruled out of the mechanism as some authors have suggested ${ }^{[1,2,4]}$.

-Reinitiations are non-determining steps according to the methodology above mentioned, however they should be considered in the modelling of these systems.

-It is very important to consider the sensitivity for the macroradical transfer in the case of furfurylidenacetone. This result is coincidental with the kinetic measurements in which furfurylidenacetone is the strongest inhibitor with the highest value of the $\mathrm{k}_{\text {trr. }}$. In fact the sensitivity analysis for the $\mathrm{k}_{\mathrm{trs}}$ follows the experimental pattern of Rp:

furfurylidenacetone $>$ furylacrolein $>$ furylacrylmorpholineamide $>5$ - methylfuranacrylic acid $>$ furfurylidenbutanal.

After the inhibition period (Table 3 ) it is necessary

Table 3. Compacted sumatory of the sensitivity coefficients of the kinetic parameters for each system after the inhibition period

\begin{tabular}{l|c|c|c|c|c|c|c|c|c|c|c}
\hline Compound & $\mathrm{f}$ & $\mathrm{kd}$ & $\mathrm{ki}$ & $\mathrm{kp}$ & $\mathrm{kt}$ & $\mathrm{ktrs}$ & $\mathrm{kis}$ & $\mathrm{krts}$ & $\mathrm{k}$ 'trs & $\mathrm{k}$ 'is & $\mathrm{k}$ 'rts \\
\hline furylacrolein & 22 & 22 & $*$ & 0 & 28 & 32 & 2.4 & 3.1 & 1.0 & 0 & 52 \\
& 12 & 12 & $*$ & 0.3 & 1.2 & 15.5 & 0.5 & 4 & 1.0 & 0 & 96 \\
furfurylidenacetone & 8 & 8 & $*$ & 0.4 & $*$ & 5.6 & 0 & 412 & 2.0 & 0 & 532 \\
furylacrylmorfolineamide & 6 & 6 & $*$ & 0.3 & $*$ & 3 & 0.3 & 3.1 & 1.4 & 0 & 410 \\
5-methylfuranacrylic ac. & 5 & 5 & $*$ & 0.6 & $*$ & 2.1 & 0 & 260 & 1.9 & 0 & 295 \\
\hline
\end{tabular}

* The sensitivity coefficient is higher than 1000 . 
to point out that:

-There is a trend to increase the values of the sensitivity coefficients after the inhibition period. The concentrations of all species, except that of the monomer, are very small. Thus a drastic change in the value of a kinetic parameter causes a notable variation of them, see Equation 1.

- The sensitivity of $\mathrm{kp}$ does not increase as expected for an unhibited polymerization. This result may be a mathemathical consequence of the fact that the propagation step does not change the concentration of the macroradicals in the system:

$$
\mathrm{M}^{*}+\mathrm{M} \longrightarrow \mathrm{M}^{*}
$$

resulting in a rather low sensitivity.

-The un-crossed termination (kt) is the most important way of termination, with the exception in the case of furfurilidenacetone for which the cross terminations are also determinant. In this case, the transfer reaction occurs to a very great extent while the reinitiations are not so important, therefore there is a high concentration of the radical MZ*

\section{Modelling}

The program to model the mechanism of polymerization was written in FORTRAN-77 and implemented for IBM-compatible microcomputer.
This program permits to model all the monomer concentrations versus time simultaneously for several runs using a GEAR algorithm to solve the stiff system of non-linear first order ordinary equations obtained from the mechanism (See Appendix 1).

The modelling of the system allows to estimate a set of kinetic parameters which minimizes the function error S (see Eq. 2), until all the values of the calculated monomer concentration (Mcal) for different runs differ less than $3 \%$ for all the experimental data (Mexp).

$$
\mathrm{S}=\Sigma\left(\mathrm{M}_{\text {exp }}-\mathrm{M}_{\text {cal }}\right)^{2}
$$

Steepest Descent ${ }^{[6]}$ was the algorithm used for the minimization.

Each system was modelled using different initial sets of the kinetic constants which are within an order of magnitude of its literature, experimental or theoretical value. All the kinetic parameters of the mechanism were allowed to improve.

The kinetic constants reported in Table 4 are the mean values obtained for those different modellings belonging to the same statistical universe according to a Fisher test ${ }^{[13]}$. facts:

It is necessary to call the attention to the following

-It is not possible to avoid in the modelling the statistical dependence of the kinetic parameters, because the system follows an intrinsic stationary state

\begin{tabular}{|c|c|c|c|c|c|c|}
\hline Inhibitor & $\mathrm{f}$ & $\mathrm{kd} .10^{6}$ & ki & kp. $10^{-3}$ & kt. $10^{-7}$ & \\
\hline furylacrolein & 0.60 & 4.1 & 15.8 & 1.32 & 2.55 & \\
\hline furfurylidenacetone & 0.62 & 1.2 & 15.4 & 2.01 & 1.00 & \\
\hline furylacrylmorfolineamide & 0.78 & 0.1 & 15.6 & 1.63 & 2.6 & \\
\hline 5-methylfuranacrylic ac. & 0.65 & 4.2 & 17 & 1.8 & 2.14 & \\
\hline hydrofuramide & 0.63 & 5.0 & 16.2 & 0.39 & 0.86 & \\
\hline Inhibitor & ktrs. $10^{-5}$ & kis. $10^{4}$ & krts. $10^{-7}$ & $\mathrm{k}^{\prime}$ is $.10^{-4}$ & k'rts. $10^{-7}$ & $\mathrm{k}^{\prime}$ rts. $10^{-5}$ \\
\hline furylacrolein & 3.1 & 4.7 & 2.9 & - & - & - \\
\hline furfurylidenacetone & 6.95 & - & 1.42 & - & - & - \\
\hline furylacrylmorfolineamide & 0.1 & - & 2.1 & 1.4 & - & 1.64 \\
\hline 5-methylfuranacrylic acid & 0.02 & 4.8 & 2.9 & 0.3 & 4 & 2.75 \\
\hline hydrofuramide & 0.01 & - & 2.1 & 1.15 & - & 0.88 \\
\hline
\end{tabular}

Table 4. Estimated kinetic parameters for the system vinyl acetate $(3.254 \mathrm{~mol} / \mathrm{L})$ - AIBN $(0.002 \mathrm{~mol} / \mathrm{L})$ - furan compound 
that creates new mathemathical relations. Thus, the sets of parameters obtained must be considered as "estimates". Better results have been obtained ${ }^{9}$ by modelling simultaneously the molecular weight and the monomer concentration. But it is not the case. Therefore, it could be convenient to have a new modelling using in the error function another independent measurement such as the molecular weight .

- The parameters $\mathrm{f}$ and $\mathrm{kd}$ are interdependent because they appear always as a product in the rate equations.

-The literature value of $\mathrm{ki}=16^{[14]}$ seems to fit well in all systems.

-The values of $\mathrm{kp}$ and $\mathrm{kt}$ are slightly smaller than the literature data. It could be due to a change of the mechanism when the polymerization occurs at low monomer concentrations ${ }^{[15]}$. In the case of hydrofuramide, its structure differs from the other compounds and other reactions can be operating.

-The reactivity order obtained from the estimated value of the $\mathrm{k}_{\mathrm{trs}}$ is the same as the order obtained from the kinetic measurements ${ }^{[8]}$. It confirms the importance of stabilization of $\mathrm{MZ}^{*}$ by conjugation on the strength of the inhibitor.

\section{Conclusions}

The radical polymerization of vinyl acetate inhibited by furan compounds could be simulated and a reliable set of rate constants for each inhibitor obtained by fitting simultaneously the monomer concentration in different kinetic runs.

The main features of the mechanism were revealed by combining these results with a sensitivity analysis.

\section{Appendix}

Rate equations for the mechanism of inhibited radical polymerization

$$
\begin{aligned}
& \frac{\mathrm{d}[\mathrm{I}]}{\mathrm{dt}}=-2 \mathrm{fkd}[\mathrm{I}] \\
& \frac{\mathrm{d}[\mathrm{M}]}{\mathrm{dt}}=-\mathrm{ki}\left[\mathrm{R}^{*}\right][M]-\mathrm{kp}[\mathrm{M}]\left[\mathrm{M}^{*}\right]-\mathrm{kis}\left[M Z^{*}\right][M]-\mathrm{k}^{\prime} \mathrm{is}\left[\mathrm{RZ}^{*}\right][\mathrm{M}] \\
& \frac{\mathrm{d}\left[\mathrm{M}^{*}\right]}{\mathrm{dt}}=-\mathrm{ki}\left[\mathrm{R}^{*}\right][\mathrm{M}]-\mathrm{kt}[\mathrm{M}]^{2}-\mathrm{ktrs}\left[\mathrm{M}^{*}\right][M]-\mathrm{ktrs}\left[\mathrm{MZ}^{*}\right]\left[\mathrm{M}^{*}\right]-\mathrm{k}^{\prime} \operatorname{rts}\left[\mathrm{RZ}^{*}\right]\left[\mathrm{M}^{*}\right]
\end{aligned}
$$

$$
\begin{aligned}
& \frac{\mathrm{d}[\mathrm{Z}]}{\mathrm{dt}}=-\mathrm{ktrs}\left[\mathrm{M}^{*}\right][\mathrm{Z}]-\mathrm{k}^{\prime} \operatorname{trs}\left[\mathrm{R}^{*}\right][\mathrm{Z}] \\
& \frac{\mathrm{d}\left[\mathrm{R}^{*}\right]}{\mathrm{dt}}=2 \mathrm{f} \mathrm{kd}[\mathrm{I}]-\mathrm{ki}^{2}\left[\mathrm{R}^{*}\right]\left[\mathrm{M}^{*}\right]-\mathrm{k}^{\prime} \operatorname{trs}\left[\mathrm{R}^{*}\right][\mathrm{Z}] \\
& \frac{\mathrm{d}\left[\mathrm{MZ} Z^{*}\right]}{\mathrm{dt}}=\mathrm{ktrs}\left[\mathrm{M}^{*}\right][\mathrm{Z}]-\mathrm{kis}\left[M Z^{*}\right][\mathrm{M}]-\mathrm{ktrs}\left[M Z^{*}\right]\left[\mathrm{M}^{*}\right] \\
& \frac{\mathrm{d}\left[\mathrm{RZ}^{*}\right]}{\mathrm{dt}}=\mathrm{k}^{\prime} \operatorname{trs}\left[\mathrm{R}^{*}\right][\mathrm{Z}]-\mathrm{k}^{\prime} \operatorname{is}\left[\mathrm{RZ}^{*}\right][\mathrm{M}]-\mathrm{k}^{\prime} \operatorname{trs}\left[\mathrm{RZ}^{*}\right]\left[\mathrm{M}^{*}\right]
\end{aligned}
$$

\section{References}

1. Kice, J. L. J. Am. Chem. Soc. 76, 6274, (1954)

2. Atkinson, W.H., Bamford, C. H., and Eastmond, G. C. Trans. Faraday Soc. 66, 1446,(1970).

3. Deb, P.C. Eur. Polym. J.16, 759, (1980).

4. Tudös, F., Foldes-Berezsnich, T., Prog. Polym. Sci. 14, 717, (1989).

5. Rieumont, J., Davidenko, N., and Paz, J.A., Acta Polym. 38, 21, (1987).

6. Rieumont, J., Vega, R. Davidenko, N., and Paz, J.A., Eur. Polym J. 24, 909, (1988).

7. Rieumont, J., Vega, R. Makromol. Chem. 192, 1387, (1991).

8. Vega, R., Rieumont, J. "Kinetic and structural features of the furan compunds as inhibitors of the radical polymerization of vinyl acetate" To be published.

9. Paz, J.A., Rieumont, J., Polymer, 36, (12), 2421, (1995).

10. Ponomarieb, A.A., "Synthesis and reactions of furan compounds." Saratov University Press. Russia. (1960).

11. Come, G. N. "Modern Methods in Kinetics; Comprehensive Chemical Kinetics" (Eds C.H. Bamford and C.F.M. Tipper), Elsevier, Amsterdam, Vol 24/3, p.249, (1983).

12. Gear, C. W. Proc. IFIP Congress, Edinburgh (ed. A. J. H. Morrell), p 187, (1968).

13. Hill, D. J. T., O’Donnell, J.H. O' Sullivan, P.W., Makromol. 18, 9, (1985).

14. Ito, K, Omi, M., Ito, T., Polym. J. 14 (2), 115, (1962).

15. Conix, A. Smets, G., J. Polym. Sc. 10, 525, (1953). 